AUTORES:

Patrícia Coutinho ${ }^{1}$

Carolina $\mathrm{Hax}^{2}$

Isabel Mesquita ${ }^{1}$

${ }^{1} \mathrm{CIFI}^{2} \mathrm{D}$, Faculdade de Desporto Universidade do Porto, Portugal

${ }^{2}$ Universidade Federal de Pelotas, Rio Grande do Sul, Brasil

https://doi.org/10.5628/rpcd.11.02.84

\section{Caracterização da prática desportiva de voleibolistas portugueses em função do nível de expertise e do género}

\author{
PALAVRAS CHAVE: \\ Desenvolvimento desportivo a longo-prazo. \\ Especialização precoce. Diversificação. \\ Natureza da prática.
}

\title{
RESUMO
}

O presente estudo pretendeu analisar a natureza da prática desportiva dos voleibolistas portugueses, tendo em consideração o seu nível de expertise e o género. A amostra deste estudo foi constituída por 60 jogadores de voleibol, sendo 30 jogadores experts e 30 jogadores não experts. Recorremos a uma entrevista retrospetiva para analisarmos a idade de iniciação no voleibol e a natureza das atividades praticadas (modalidades individuais e coletivas) ao longo de 3 etapas de formação desportiva (6-12 anos, 13-16 anos e 17-20 anos). Os resultados demonstraram que: 1) os voleibolistas portugueses possuem perfis semelhantes no que se refere à idade de iniciação no voleibol, independentemente do nível de expertise e do género; 2) os jogadores praticaram mais modalidades individuais e coletivas na etapa 1 e 2; e 3) as mulheres praticaram mais modalidades individuais durante a sua formação do que os homens. Este estudo demonstrou que a idade de iniciação no voleibol e a natureza das atividades praticadas não se constituíram fatores influenciadores do alcance da expertise dos voleibolistas portugueses. No entanto, o género mostrou perfis diferenciados no que concerne à natureza da prática porquanto as mulheres praticaram mais desportos individuais do que os homens. Este aspeto fornece informação relevante para a construção de programas de desenvolvimento desportivo, sendo que o género deve ser uma variável a considerar na definição dos percursos de desenvolvimento da carreira desportiva dos jogadores de Voleibol. 


\section{volleyball players according}

\section{to expertise level and gender}

\section{ABSTRACT}

This study aimed to analyse the sport participation of Portuguese volleyball players, according to their expertise level and gender. The sample of this study included 60 volleyball players, with 30 experts and 30 nonexperts players. From a retrospective interview, we examined the volleyball starting age and the nature of activities practiced (i.e. individual and team sports) throughout 3 developmental stages considered (6-12 years, 13-16 years and 17-20 years). The results of this study showed that: 1) the Portuguese volleyball players had similar volleyball starting ages, independently of expertise level and gender; 2) the volleyball players have practiced more individual and team sports during stage 1 and stage 2; and 3) female players have practiced more individual sports during their sport development than male players. This study showed that the volleyball starting age and the nature of the activities practiced were not key factors in the expertise achievement of Portuguese Volleyball Players. However, a different sport participation profile according to the gender was found since female players practiced more individual sports than male players. This result offers valuable information to the sport programmes development as gender is a variable that should be considered to distinguishes the pathways of the Volleyball players throughout their sportive career.

\section{KEY WORDS:}

Long-term athlete development. Early specialization. Early diversification. Nature of practice. 


\section{INTRODUÇÃo}

O estudo do processo de aquisição e manifestação de aptidões desportivas, físicas, cognitivas e psicológicas que conduzem ao alcance da expertise tem sido alvo de elevada atenção

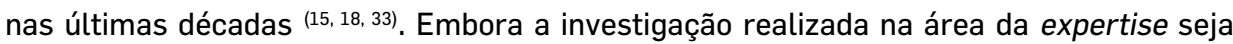
dominada pelo célebre debate nature-nurture, onde os investigadores se dividem entre a importância dos fatores genéticos ou ambientais para o alcance da expertise, recentemente tem sido proclamada a necessidade de uma visão multifatorial e integradora destes fatores $(17,27)$. Os fatores relacionados com o envolvimento desportivo, nomeadamente, o papel da prática (i.e a quantidade e tipo) no desenvolvimento e alcance da expertise têm preenchido grande parte da agenda da investigação ${ }^{(8 ., 10,25)}$.

A teoria da prática deliberada ${ }^{(18)}$ tem sido uma das referências teóricas mais influentes nos estudos que analisam a importância da quantidade e do tipo de prática desportiva. De acordo com os autores, a prática deliberada caracteriza-se como uma atividade altamente estruturada, que requer esforço físico e cognitivo e pode não ser intrinsecamente motivante; concorrente do grande objetivo de desenvolver e aumentar a performance. Adicionalmente, esta teoria sugere que são necessários 10 anos ou 10.000 horas de prática específica para se alcançar um patamar elevado de rendimento em qualquer contexto de realização. Embora originalmente desenvolvida em pesquisas centradas na música, diversos autores têm testado e sugerido a sua aplicabilidade no desporto (11, 18, 21, 22, 23).

A expansão da investigação da teoria da prática deliberada aplicada ao contexto desportivo tem permitido atestar que nem todos os pressupostos desta teoria são necessários para o alcance da expertise ${ }^{(3,4)}$. Diversos estudos têm vindo a demonstrar que os atletas que obtiveram uma vivência desportiva inicial diversificada, com predominância do jogo deliberado (i.e., atividade intrinsecamente motivante, que promove uma gratificação imediata e é intencionalmente concebida para maximizar o divertimento) ${ }^{(15)}$, alcançaram de igual forma elevados níveis de rendimento ${ }^{(6,7,10)}$.

Constatando a possibilidade da existência de diversos percursos para o alcance da expertise, Côté e colaboradores ${ }^{(12,13,15)}$ desenvolveram e aprimoraram o denominado Modelo Desenvolvimental de Participação no Desporto (MDPD) ao longo da última década. Assente no postulado de promover não só a performance, mas igualmente uma participação desportiva sustentada e um desenvolvimento pessoal favorável, o respetivo modelo sugere a existência de três percursos desportivos possíveis: 1) participação recreativa através da diversificação desportiva e do jogo deliberado; 2) alcance da expertise através da diversificação desportiva e do jogo deliberado; e 3) alcance da expertise através da especialização precoce e da prática deliberada. No que se refere à trajetória da diversificação desportiva e jogo deliberado (2), o modelo estabelece ainda três etapas distintas que os atletas devem percorrer: i) anos de Experimentação (6-12 anos); ii) anos de Especialização (13-16 anos), e iii) anos de Investimento (+16 anos). De acordo com os autores, a participação despor- 
tiva vai decrescendo desde os anos de experimentação (i.e., prática de muitas atividades desportivas) até aos anos de investimento (i.e., focalização em apenas um desporto).

0 desenvolvimento e aplicação extensiva do MDPD pela investigação ${ }^{(8,10,24,29)}$ tem demonstrado que o número de horas de treino que os atletas acumulam ao longo da sua carreira influencia o alcance da expertise. Relativamente ao tipo de prática, esta variável é considerada como um dos principais fatores no desenvolvimento da expertise ${ }^{(16,18,34)}$, pelo que os investigadores têm vindo a caracterizar com pormenor a especificidade da prática (específica ou não específica) e o seu nível de formalidade (estruturada e não estruturada). Conscientes da possibilidade de transferência de aprendizagens entre a prática de diferentes atividades ${ }^{(1,7)}$, alguns autores têm vindo a analisar a contribuição específica da prática estruturada concretizada pelo atleta numa fase inicial da sua carreira para o alcance da expertise ${ }^{(25)}$. Todavia, não se tem indagado acerca da influência da prática de atividades estruturadas com características distintas do desporto principal no alcance da expertise. Particularmente no caso dos jogos desportivos coletivos, desconhece-se o papel da prática de desportos individuais numa fase inicial do desenvolvimento do atleta para o potenciamento das suas capacidades e, consequentemente, para o alcance de elevados níveis de rendimento.

Acrescenta-se, ainda, que a maioria dos estudos desenvolvidos na área do desenvolvimento do talento e da expertise recorre a amostras constituídas por atletas masculinos, pelo que a análise de atletas femininos, bem como a comparação entre a prática desportiva de ambos, tem sido amplamente descurada. De facto, pouco se sabe sobre quais são as especificidades de cada género relativamente aos padrões de participação desportiva e à sua influência no desenvolvimento da expertise.

Tendo em consideração o anteriormente exposto, constitui-se como objetivo deste estudo: 1) caracterizar a participação desportiva dos voleibolistas portugueses (i.e., a idade de iniciação no voleibol, o número e a natureza das atividades praticadas) ao longo das diferentes etapas de formação; e 2) averiguar se os padrões de participação desportiva ao longo do tempo variam em função do género e do nível de expertise dos atletas.

\section{METODOLOGIA}

AMOSTRA

A amostra deste estudo compreendeu 30 atletas experts e 30 atletas não-experts, sendo cada grupo composto por 15 atletas do género feminino e 15 atletas do género masculino. Os 60 atletas que participaram neste estudo foram selecionados tendo por base os seguintes critérios: a) ter uma idade igual ou superior a 23 anos, e b) possuir no mínimo 10 anos de prática de voleibol. Adicionalmente, os atletas experts teriam de possuir os seguintes critérios: a) jogarem na divisão nacional mais competitiva (primeira divisão); b) terem par- 
ticipado na seleção nacional sénior, e c) serem considerados pelos treinadores da seleção nacional como sendo dos melhores jogadores nacionais de voleibol. Relativamente aos atletas não-experts, estes foram selecionados por a) jogarem numa divisão competitiva inferior ( $2^{a}$ ou $3^{a}$ divisão), e b) nunca terem participado na seleção nacional (cadetes, juniores ou seniores). Os atletas foram contactados pessoalmente ou por via telefónica, tendo-lhes sido proporcionada uma visão global do estudo. A percentagem de concordância para participar no estudo foi de $100 \%$. As entrevistas foram conduzidas pela primeira autora num ambiente calmo e silencioso, de forma a dispor das melhores condições possíveis para recolher a informação desejada sobre o percurso desportivo dos atletas. Previamente ao início da entrevista, os atletas assinaram um termo de consentimento e analisaram um folheto informativo fornecido sobre o objetivo do estudo e os procedimentos inerentes à entrevista. A realização das entrevistas teve uma duração de 45 minutos a 2 horas.

\section{INSTRUMENTO E PROCEDIMENTOS}

No sentido de recolhermos informação relativa ao percurso desportivo dos voleibolistas portugueses, foi construída e aplicada uma versão adaptada da entrevista retrospetiva desenvolvida por Côté, Ericsson e Law ${ }^{(14)}$. A entrevista compreendia 10 questões fechadas que intentavam recolher informação sobre: a) dados demográficos do atleta (i.e., idade, género, nível competitivo), b) idade de início da prática de voleibol federativo, c) número de modalidades coletivas praticadas; e d) número de modalidades individuais praticadas. A informação relativa ao tipo de prática realizada durante o desenvolvimento do atleta foi registada em diversas tabelas e quadros que foram intencionalmente construídos para facilitar ao entrevistado a recordação da sua prática desportiva passada.

O MDPD ${ }^{(12,13,15)}$ foi utilizado como referência teórica para definir as etapas de formação desportiva consideradas neste estudo. Adicionalmente, o sistema competitivo da Federação Portuguesa de Voleibol foi considerado para uma definição mais rigorosa e contextualizada dos valores de corte relativos às idades de cada etapa. Neste sentido, foram definidas 3 etapas de formação desportiva: 6-12 anos (etapa 1), 13-16 anos (etapa 2), e 17-20 anos (etapa 3). A primeira etapa (6-12 anos) corresponde exatamente à primeira etapa considerada no MDPD. Em particular no voleibol, este grupo de idades é caracterizado pela prática informal da modalidade, pela implementação de jogos reduzidos ( $2 \times 2$ e 4x4), em que a iniciação desportiva e o divertimento constituem os principais objetivos. A segunda etapa (13-16 anos) enquadra-se dentro da segunda etapa considerada no MDPD (i.e. 13-15 anos). No voleibol, este grupo etário caracteriza-se pela introdução do jogo formal (6x6), pela implementação de uma prática mais sistemática, pelo inicio da especialização táctica e funcional, e, ainda, pelo aumento da quantidade do número e horas de treino. Finalmente, a terceira etapa (17-20 anos) integra-se dentro da terceira etapa considerada no MDPD 
(i.e., +16 anos). Particularmente no voleibol, este grupo de idades corresponde à transição júnior-sénior, quando a preparação e a implementação de elevados níveis de prática são potenciadas com o grande intuito de maximizar a performance.

\section{VALIDADE E FIABILIDADE DOS DADOS}

A análise retrospetiva constitui-se uma metodologia altamente dependente da validade da informação reportada ${ }^{(14)}$. Enquanto que relembrar eventos recentes se constitui uma tarefa relativamente fácil e confiável, recordar informações sobre atividades realizadas numa fase inicial do percurso desportivo do atleta poderá não ser uma tarefa com o mesmo índice de precisão ${ }^{(14)}$. Neste sentido, de forma a assegurarmos a fiabilidade dos dados do presente estudo, $10 \%$ da amostra foi convidada a refazer a entrevista 6 meses depois da primeira concretização. A correspondência entre os dados reportados pelos atletas nos diversos momentos foi examinada através da percentagem de concordância ${ }^{(2)}$. A fiabilidade do teste-reteste foi assegurada pelo indicador Kappa de Cohen (valores entre .82 e 1.00), o que indica que os dados deste estudo são válidos e fiáveis ${ }^{(28)}$.

\section{ANÁLISE DOS DADOS}

Para a análise quantitativa dos dados foi utilizado o programa SPSS, versão 20.0. Para a descrição dos dados foram utilizados os valores da média e desvio padrão. 0 teste Kolmogorov-Smirnov foi usado para testar a normalidade da distribuição. A análise inferencial relativa às diferenças estatísticas entre as variáveis consideradas neste estudo foi efetuada através da ANOVA de medidas repetidas com teste post-hoc para comparações múltiplas. 0 nível de significância estabelecido foi de $p<.05$.

\section{RESULTADOS}

IDADE DE INICIAÇÃO NO VOLEIBOL

Relativamente à idade de iniciação no voleibol, o perfil dos voleibolistas não se discriminou, uma vez que não se observam diferenças estatisticamente significativas para o nível de expertise $\left(\mathrm{F}_{(1,56)}=1.17, \mathrm{p}=.895\right)$ ou género $\left(\mathrm{F}_{(1,56)}=1.633, \mathrm{p}=.206\right)$. 


\begin{tabular}{|c|c|c|c|c|c|c|c|c|}
\hline & $\begin{array}{l}n \\
\frac{n}{x} \\
\frac{1}{w} \\
\frac{a}{x} \\
w\end{array}$ & 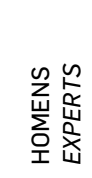 & 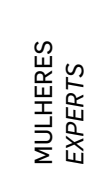 & 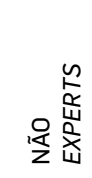 & 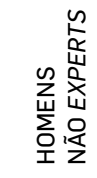 & 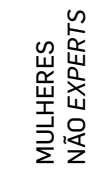 & 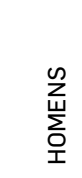 & 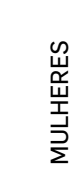 \\
\hline $\mathrm{N}$ & 30 & 15 & 15 & 30 & 15 & 15 & 30 & 30 \\
\hline média & 10.50 & 9.47 & 11.53 & 10.60 & 10.67 & 10.53 & 10.07 & 11.03 \\
\hline desvio-padrão & 3.2 & 3.6 & 2.5 & 2.7 & 3.4 & 2.0 & 3.5 & 2.3 \\
\hline
\end{tabular}

\section{NATUREZA DAS ATIVIDADES (MODALIDADES COLETIVAS E INDIVIDUAIS)}

Os voleibolistas portugueses apresentam perfis diferentes relativamente à natureza da prática vivenciada. Relativamente às modalidades individuais (MI), observam-se diferenças estatisticamente significativas em função das etapas de formação $\left(F_{(2,112)}=27.61\right.$, $\mathrm{p}<.001)$ e do género dos praticantes $\left(F_{(2,112)}=8.02, p=.001\right)$. Enquanto que, de uma forma geral, os jogadores praticaram mais modalidades individuais na etapa 1 ( $p=.001$ ) e na etapa $2(p<.001)$, as raparigas, em particular, praticaram mais MI do que os rapazes na etapa $1(p=.010)$. Não se observaram diferenças estatisticamente significativas em função do nível de expertise relativamente à prática de MI.

No que se refere à prática de modalidades coletivas (MC), observam-se diferenças estatisticamente significativas em função das etapas de formação $\left(F_{(2,112)}=7.72, p=.001\right)$, sendo que os voleibolistas, em geral, praticaram mais modalidades coletivas na etapa 1 $(p<.001)$ e na etapa $2(p<.001)$. Não se observaram diferenças estatisticamente significativas em função do nível de expertise e do género no que se refere à prática de MC.

TABELA 2 - Estatística descritiva para o número de modalidades individuais e

\begin{tabular}{|c|c|c|c|c|c|c|c|c|c|}
\hline & 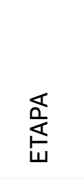 & 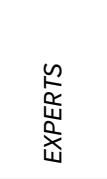 & 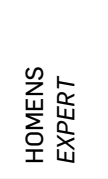 & 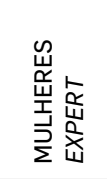 & 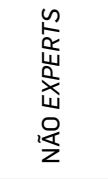 & 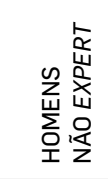 & 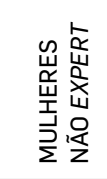 & 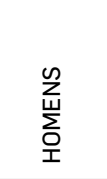 & 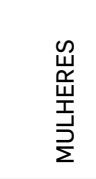 \\
\hline \multirow{3}{*}{ MI } & $6-12$ & $1.3(0.7)$ & $0.9(0.6)$ & $1.6(0.7)$ & $1.4(1.2)$ & $0.7(0.5)$ & $2.1(1.3)$ & $0.8(0.6)$ & $1.8(1.0)$ \\
\hline & $13-16$ & $1.0(0.7)$ & $0.8(0.5)$ & $1.1(0.8)$ & $0.9(1.3)$ & $0.7(0.9)$ & $1.1(1.6)$ & $0.8(0.7)$ & $1.1(1.2)$ \\
\hline & $17-20$ & $0.5(0.7)$ & $0.5(0.5)$ & $0.6(0.9)$ & $0.4(0.8)$ & $0.4(0.8)$ & $0.5(0.9)$ & $0.4(0.6)$ & $0.6(0.9)$ \\
\hline
\end{tabular}




\begin{tabular}{|c|c|c|c|c|c|c|c|c|c|}
\hline & 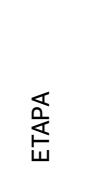 & $\begin{array}{l}\frac{w}{a} \\
\frac{x}{w} \\
\frac{a}{x} \\
w\end{array}$ & 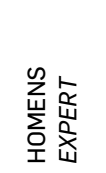 & 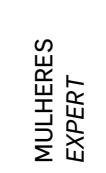 & 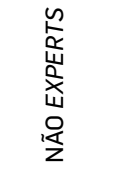 & 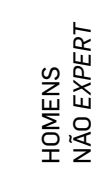 & 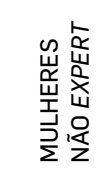 & $\begin{array}{l}\sum_{\text {W }}^{n} \\
\text { 오 }\end{array}$ & 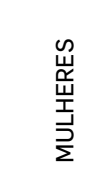 \\
\hline \multirow{3}{*}{ MC } & $6-12$ & $1.7(1.1)$ & $2.1(1.2)$ & $1.3(0.9)$ & $1.4(1.1)$ & $1.3(0.8)$ & $1.6(1.3)$ & $1.7(1.1)$ & $1.4(1.1)$ \\
\hline & $13-16$ & $2.0(0.9)$ & $2.3(1.0)$ & $1.8(0.7)$ & $2.0(1.1)$ & $1.9(1.0)$ & $2.1(1.3)$ & $2.1(1.0)$ & $1.9(1.0)$ \\
\hline & $17-20$ & $1.9(0.7)$ & $2.0(0.9)$ & $1.8(0.6)$ & $2.1(0.9)$ & $2.2(0.7)$ & $2.1(1.1)$ & $2.1(0.8)$ & $1.9(0.8)$ \\
\hline
\end{tabular}

\section{DISCUSSÃO}

Com este estudo pretendeu-se caracterizar a participação desportiva dos voleibolistas portugueses, particularmente a idade de iniciação no voleibol, a quantidade (número de modalidades praticadas) e a natureza das atividades praticadas (modalidades coletivas e individuais) ao longo de três etapas de formação desportiva, considerando o seu nível de expertise e género.

A idade de iniciação no voleibol não se diferenciou em função do nível de expertise e do género, pelo que ter iniciado a prática da modalidade precocemente (i.e., especialização precoce) ou tardiamente (i.e., diversificação) não parece ter influenciado o alcance de elevados níveis de rendimento entre os voleibolistas portugueses. Esta variabilidade de percursos vem reforçar os dados encontrados pelo estudo de Leite et al. ${ }^{(25)}$ com atletas portugueses de futebol $(n=42)$, basquetebol $(n=37)$, hóquei patins $(n=19)$ e voleibol $(n=14)$, acrescentando informação proveitosa para o voleibol português uma vez que a dimensão amostral neste estudo $(n=60)$ é consideravelmente superior. Importa, todavia, salientar que a especialização precoce tem sido associada a diversas consequências negativas, nomeadamente a ocorrência de uma maior número de lesões, decréscimo do divertimento associado à prática desportiva e, ainda, ao abandono desportivo precoce ${ }^{(3,4,32)}$.

No que concerne à natureza das atividades praticadas (i.e., modalidades coletivas e individuais), os resultados deste estudo demonstraram que a natureza da prática desportiva não influenciou o nível de expertise dos voleibolistas portugueses. Este resultado contraria a tendência vigente na literatura, onde diversos autores proclamam a importância não só de uma prática diversificada durante os primeiros anos do desenvolvimento do atleta ${ }^{(5,9,15,20)}$, mas também as vantagens da prática de atividades que apresentem características semelhantes ao desporto principal para o alcance da expertise ${ }^{(7,10)}$. Uma possível explicação para este resultado pode-se encontrar na ausência de planeamentos desportivos a longo-prazo em Portugal, o que consequentemente coloca a responsabilidade e as decisões acerca da participação desportiva para os atletas e para os pais. Isto sugere que as oportunidades desportivas disponíveis são geradas fortuitamente, o que promove o aparecimento de percursos desportivos aleatórios. Contudo, importa destacar a prática acrescida de atividades organizadas durante 
a primeira e a segunda etapa (i.e., entre os 6 e os 16 anos) pela generalidade dos atletas, 0 que vem demonstrar que, para além do desporto principal, os voleibolistas vivenciaram outros desportos na etapas iniciais da sua formação. Diversos estudos têm vindo a reconhecer os benefícios de uma prática diversificada inicial ${ }^{(3,4,20,29)}$, não só pelo potenciamento da motivação intrínseca através do divertimento e das experiências diversas que o atleta vivencia, como também pela possibilidade de adaptações cognitivas e fisiológicas que podem ser transferíveis para o desporto principal. Neste sentido, os programas desportivos devem considerar os pressupostos acima citados de forma a garantir que os jovens atletas prossigam percursos desportivos apropriados e mantenham uma ligação prolongada com o desporto.

Um dos resultados mais interessantes neste estudo prende-se com o facto de a natureza da prática ter sido um factor diferenciador do género, tendo as mulheres praticado mais modalidades individuais do que os homens. Este resultado evidencia distintas oportunidades e escolhas de prática desportiva entre homens e mulheres, o que poderá dever-se, mais uma vez, à falta de planeamento a longo prazo relativamente à participação desportiva de jovens em Portugal. Por outro lado, o estereótipo que associa a prática de modalidades individuais a uma prática menos invasiva, e por isso mais feminina, poderá ajudar-nos a compreender estes resultados. De facto, a inexistência de um quadro teórico capaz de explicar as diferenças de género limita-nos a nossa compreensão e discussão acerca deste resultado ${ }^{(26)}$, mas, por outro lado, reforça a necessidade de investigações futuras que examinem estas diferenças e que, consequentemente, auxiliem uma organização apropriada de programas desportivos.

Finalmente, importa salientar que apesar da metodologia adotada (i.e., entrevistas retrospetivas) estar limitada ao nível da precisão da memória dos atletas relativamente à sua prática desportiva passada ${ }^{(14,30)}$, este estudo proporcionou perceções importantes sobre a participação desportiva dos voleibolistas portugueses. Neste sentido, os resultados deste estudo, particularmente as diferenças existentes na participação desportiva de homens e mulheres, fornecem indicadores relevantes para a construção e desenvolvimento de planeamentos de desenvolvimento desportivo a longo-prazo no Voleibol em Portugal. Adicionalmente, investigações futuras devem procurar analisar com maior rigor e detalhe a microestrutura da prática, no sentido de proporcionar uma melhor compreensão sobre as atividades que mais contribuem para o alcance da expertise ${ }^{(19,31)}$.

\section{CONCLUSÕES}

Os resultados deste estudo demonstraram que a natureza das atividades praticadas foi distinta entre os homens e as mulheres, tendo estas últimas praticado mais modalidades individuais numa fase inicial da sua carreira desportiva, o que evidencia diferentes experiências e oportunidades desportivas. Por outro lado, o nível de expertise dos voleibolistas portugueses não foi diferenciado pela idade de iniciação no voleibol e pela natureza da 
atividades praticadas, o que incita a necessidade de percebermos o que terá contribuído para o alcance da expertise entre os voleibolistas portugueses. Neste sentido, embora as entrevistas retrospetivas constituam uma importante ferramenta para analisarmos a perceção dos atletas acerca da sua prática desportiva passada, esta área de investigação deve avançar para caracterizações mais pormenorizadas da prática (i.e. microestrutura da prática), recorrendo consequentemente a observações sistemáticas da prática e/ ou à aplicação de estudos longitudinais que possam cumprir esta função. A obtenção de um conhecimento detalhado sobre as experiências desportivas dos voleibolistas portugueses poderá contribuir significativamente para a construção e desenvolvimento de programas de desenvolvimento desportivo a longo prazo.

\section{AGRADECIMENTOS}

Este trabalho foi financiado pela Fundação para a Ciência e a Tecnologia (FCT) - Portugal (SFRH/ BD/ 64680/ 2009) no âmbito do POPH/ QREN co-financiado pelo Fundo Social Europeu (FEDER). 
1. Abernethy B, Baker J, Côté J (2005). Transfer of Pattern Recall Skills May Contribute to the Development of Sport Expertise. Applied Cognitive Psychology 19, 6: 705-18.

2. Bahrick HP, Hall LK, Berger SA (1996). Accuracy and distortion in memory for high school grades. Psychol Sci 7: 265-71.

3. Baker J (2003). Early Specialization in Youth Sport: a requirement for adult expertise? High Ability Studies 14(1): 85-94.

4. Baker J, Cobley S, Fraser-Thomas J (2009). What do we know about early sport specialization? Not much! High Ability Studies 20 (1): 77-89.

5. Baker J, Côté J (2006).Shifting training requirements during athlete development: Deliberate practice, deliberate play and other sport involvement in the acquisition of sport expertise. In: Tenenbaum DHG (ed.). Essential processes for attaining peak performance. Oxford: Meyer \& Meyer Sport; p. 92-109.

6. Baker J, Côté J, Abernethy B (2003a). Learning From the Experts: Practice Activities of Expert Decision Makers in Sport. Res Q Exerc Sport 74 (3) : 342-7.

7. Baker J, Côté J, Abernethy B (2003b). Sport-Specific Practice and the Development of Expert Decision-Making in Team Ball Sports. Journal of Applied Sport Psychology 15: 12-25.

8. Baker J, Côté J, Deakin J (2005b). Expertise in Ultra-Endurance Triathletes Early Sport Involvement, Training Structure, and the Theory of Deliberate Practice. Journal of Applied Sport Psychology 17: 64-78.

9. Baker J, Côté J, Deakin J (2006). Patterns of Early Involvement in Expert and Nonexpert Master Triathletes. Res Q Exerc Sport 77 (3): 401-7.

10. Berry J, Abernethy B, Côté J (2008). The Contribution of Structured Activity and Deliberate Play to the Development of Expert Perceptual and Decision-Making Skill. Journal of Sport \& Exercise Psychology 30: 685-708.

11. Charness N, Tuffiash M, Krampe R, Reingold E, Vasyukova E (2005). The Role of Deliberate Practice in Chess Expertise. Applied Cognitive Psychology 19 (2): 151-65.
12. Côté J, Baker J, Abernethy B (2003).From play to practice: A developmental framework for the acquisition of expertise in team sport. In: Starkes J, Ericsson KA (ed.). Expert performance in sports: Advances in research on sport expertise. Champaign, IL: Humam Kinetics; p. 89-113.

13. Côté J, Baker J, Abernethy B (2007).Practice and Play in the development of Sport Expertise. In: Eklund R, Tenenbaum G (ed.). Handbook of Sport Psychology. 3rd ed. Hoboken, NJ: Wiley; p. 184-202.

14. Côté J, Ericson KA, Law M (2005). Tracing the Development of Athletes Using Retrospective Interview Methods: A Proposed Interview and Validation Procedure for Reported Information. Journal of Applied Sport Psychology 17: 1-19.

15. Côté J, Murphy-Mills J, Abernethy B (2012).The development of skill in sport. In: Hodges N, Williams AM (ed.). Skill acquisition in sport - research, theory and practice. New York: Routledge.

16. Davids K (2000). Skills Acquisition and the Theory of Deliberate Practice: It Ain't What You Do It's the Way That You Do It. International Journal of Sport $\&$ Exercise Psychology 31: 461-6.

17. Davids K, Baker J (2007). Genes, environment and sport-performance: why the nature-nurture dualism is no longer relevant. Sports Med 37 (11): 1-20.

18. Ericsson K, Krampe R, Tesch-Romer C (1993). The Role of Deliberate Practice in the Acquisition of Expert Performance. Psychol Rev 100 (3): 363-406.

19. Ford P, Yates I, Williams AM (2010). An analysis of practice activities and instructional behaviours used by youth soccer coaches during practice: Exploring the link between science and application. J Sports Sci, 28 (5): 483-95.

20. Fraser-Thomas J, Côté J, Deakin J (2008). Examining Adolescent Sport Dropout and Prolonged Engagement from a Developmental Perspective. Journal of Applied Sport Psychology 20 (3): 318-33.

21. Helsen WF, Hodges NJ, Winckel J, Starkes J (2000). The roles of talent, physical precocity and practice in the development of soccer expertise. Journal of Sport Sciences 18: 727-36. 


\section{5}

22. Helsen WF, Starkes J, Hodges N (1998). Team Sports and the Theory of Deliberate Practice. Journal of Sport \& Exercise Psychology 20: 12-34.

23. Hodge T, Deakin J (1998). Deliberate Practice and Expertise in the Martial Arts: The Role of Context in Motor Recall. Journal of Sport \& Exercise Psychology 20: 260-79.

24. Law M, Côté J, Ericsson KA (2007). Characteristics of Expert Development in Rhythmic Gymnastics: A Retrospective Study. International Journal of Sport \& Exercise Psychology 5: 82-103.

25. Leite N, Baker J, Sampaio J (2009). Paths to expertise in Portuguese national team athletes. Journal of Sports Science and Medicine, 8 (4): 560-6.

26. Leite N, Sampaio J (2012). Long-Term Athletic Development Across Different Age Groups and Gender from Portuguese Basketball Players. International Journal of Sports Science \& Coaching, 7 (2): 285-300.

27. Phillips E, Davids K, Renshaw I, Portus M (2010). Expert performance in Sport and the dynamics of talent development. Sports Med, 40 (4): 271-83.

28. Reiss D (1981). Family's constrution of reality. MA: Harvard University Press.

29. Soberlack P, Côté J (2003). The Developmental Activities of Elite Ice Hockey Players. Journal of Applied Sport Psychology 15: 41-9.

30. Sosniak LA (2006). Retrospective Interviews in the Study of Expertise and Expert Performance. In: Ericsson KA, Charness N, Feltovich PJ, Hoffman R (ed.). The Cambridge Handbook of Expertise and Expert Performance. New York: Cambridge University Press; p. 287-301.

31. Ward P, Hodges N, Starkes J, Williams AM (2007). The road to excellence: deliberate practice and the development of expertise. High Ability Studies 18 (2): 119-153.

32. Wiersma LD (2000). Risks and Benefits of Youth Sport Specialization: Perspectives and Recommendations. Pediatric Exercise Science 12: 13-22.

33. Williams AM, Ford $P$ (2008). Expertise and expert performance in sport. International Review of Sport and Exercise Psychology, 1 (1): 4-18.
34. Williams AM, Hodge NJ (2005). Practice, instruction and skill acquisition in soccer: challenging tradition. Journal of Sports Science, 23: 637-50. 